\title{
The X-linked mental retardation gene PHF8 is a histone demethylase involved in neuronal differentiation
}

\author{
Jihui Qiu ${ }^{2, *}$, Guang Shi ${ }^{1, *}$, Yuanhui Jia ${ }^{1, *}$, Jing $\mathrm{Li}^{1}$, Meng Wu ${ }^{1}$, Jiwen $\mathrm{Li}^{1}$, Shuo Dong ${ }^{2}$, Jiemin Wong ${ }^{1}$ \\ ${ }^{I}$ The Institute of Biomedical Sciences and School of Life Sciences, East China Normal University, 500 Dongchuan Road, Shanghai \\ 200241, China; ${ }^{2}$ Department of Medicine, Baylor College of Medicine, Houston, TX 77030, USA
}

Recent studies have identified mutations in PHF8, an X-linked gene encoding a JmjC domain-containing protein, as a causal factor for X-linked mental retardation (XLMR) and cleft lip/cleft palate. However, the underlying mechanism is unknown. Here we show that PHF8 is a histone demethylase and coactivator for retinoic acid receptor (RAR). Although activities for both $\mathrm{H3K} 4 \mathrm{me} / 2 / 1$ and $\mathrm{H3K} 9 \mathrm{me} / 1$ demethylation were detected in cellularbased assays, recombinant PHF8 exhibited only H3K9me2/1 demethylase activity in vitro, suggesting that PHF8 is an H3K9me2/1 demethylase whose specificity may be modulated in vivo. Importantly, a mutant PHF8 (phenylalanine at position 279 to serine) identified in the XLMR patients is defective in enzymatic activity, indicating that the loss of histone demethylase activity is causally linked with the onset of disease. In addition, we show that PHF8 binds specifically to H3K4me3/2 peptides via an N-terminal PHD finger domain. Consistent with a role for PHF8 in neuronal differentiation, knockdown of PHF8 in mouse embryonic carcinoma P19 cells impairs RA-induced neuronal differentiation, whereas overexpression of the wild-type but not the F279S mutant PHF8 drives P19 cells toward neuronal differentiation. Furthermore, we show that PHF8 interacts with RAR $\alpha$ and functions as a coactivator for RAR $\alpha$. Taken together, our results suggest that histone methylation modulated by PHF8 plays a critical role in neuronal differentiation.

Keywords: PHF8; histone demethylase; coactivator; XLMR; neuronal differentiation

Cell Research (2010) 20:908-918. doi:10.1038/cr.2010.81; published online 15 June 2010

\section{Introduction}

Precise gene expression is essential for correct cellular identity and differentiation in mammalian development $[1,2]$. As the eukaryotic genome is assembled into chromatin, histone modifications including phosphorylation, acetylation and methylation govern chromatin dynamics and are critical determinants of gene expression [3].

Among various types of histone modifications, histone lysine methylation has been extensively linked with gene activation and gene repression [4]. Multiple lysine residues in different histone subunits can be methylated

*These three authors contributed equally to this work

Correspondence: Jiemin Wong ${ }^{\mathrm{a}}$, Shuo Dong ${ }^{\mathrm{b}}$

${ }^{\mathrm{a}}$ Tel: +86-21-54345013; Fax: +86-21-54344922

E-mail: jmweng@bio.ecnu.edu.cn

${ }^{b}$ E-mail: sdong@bcm.edu

Received 28 April 2010; revised 7 May 2010; accepted 8 May 2010; published online 15 June 2010 and methylation is catalyzed by a family of SET domaincontaining methyltransferases [5]. As another layer of complexity, methylation on lysine can occur in three different states: mono-, di- and tri-methylation. Histone methylation influences transcription in a site- and statedependent manner [4]. For instance, H3K $4 \mathrm{me} 3 / 2$ and $\mathrm{H} 3 \mathrm{~K} 9 \mathrm{me} 3 / 2$ at gene promoters have been generally correlated with active transcription and gene silencing, respectively. Mechanistically, H3K4me3/2 has been shown to recruit the general transcription factor TFIID [6] and the nucleosome remodeling factor NURF [7] and to inhibit the binding of the NURD corepressor complex $[8,9]$. On the other hand, H3K9me $3 / 2$ serves as a binding site for heterochromatin protein 1 (HP1) and its associated repressor proteins $[10,11]$.

Although histone methylation was not long ago thought to be irreversible due to the high thermodynamic stability of the N-CH3 bond, the identification of LSD1 and subsequent JmjC family demethylases changed this dogma $[12,13]$. While LSD1 catalyzes lysine demethy- 
lation via an FAD-dependent oxidation reaction, the JmjC family demethylases remove the methyl moiety through a hydroxylation reaction and utilize iron and $\alpha$-ketoglutarate as cofactors $[14,15]$. The conserved $\mathrm{JmjC}$ domain comprises the enzymatic core of these family enzymes [13].

PHF8 is an X-linked gene encoding an evolutionarily conserved protein containing an N-terminal PHD (plant homeodomain) domain and a JmjC domain, the latter being a signature of JmjC family histone demethylases. Nonsense and missense mutations in PHF8 have been causally linked with X-linked mental retardation (XLMR) with cleft lip/cleft palate $[16,17]$. However, the underlying mechanism is not known.

Here we show that PHF8 is a histone demethylase, and importantly, an F279S mutant identified in PHF8 from a family of XLMR patients is deficient in enzymatic activity [17]. In addition, we show that PHF8 interacts with and functions as a coactivator for retinoic acid receptor
(RAR). Knockdown of PHF8 in mouse embryonic carcinoma P19 cells impairs RA-induced neuronal differentiation, whereas overexpression of the wild-type but not the F279S mutant PHF8 drives P19 cells toward neuronal differentiation. Our results suggest that histone demethylation regulated by PHF8 plays a critical role in neuronal differentiation.

\section{Results}

PHF8 exhibits both H3K4 and H3K9 demethylase activity in cells

Given the presence of a JmjC signature domain for histone demethylase activity (Figure 1A), we wished to examine if PHF8 possesses histone demethylase activity. We first ectopically expressed Flag-tagged PHF8 in HeLa cells and examined demethylase activity by immunofluorescence using antibodies specific for various methylated histones. Among various sites and methyla-
A

PHF8

PHF8 (H247A)

PHF8 (F279S)

PHF8 ( $\triangle \mathrm{PHD})$

B

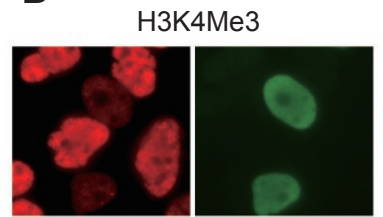

$\mathrm{H} 3 \mathrm{~K} 4 \mathrm{Me} 3$

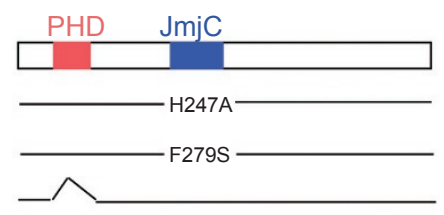

C

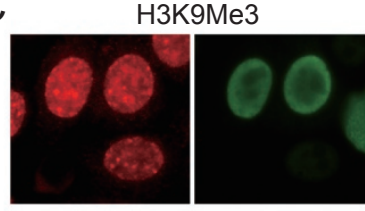

$\mathrm{H} 3 \mathrm{~K} 9 \mathrm{Me} 2$

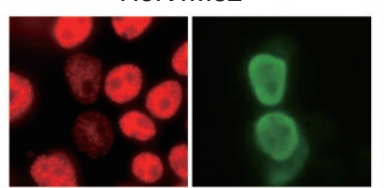

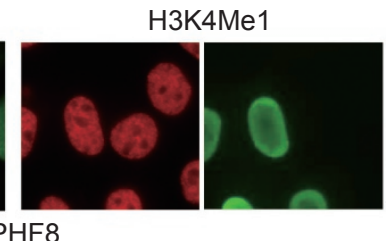

PHF8

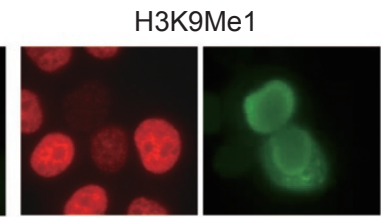

PHF8
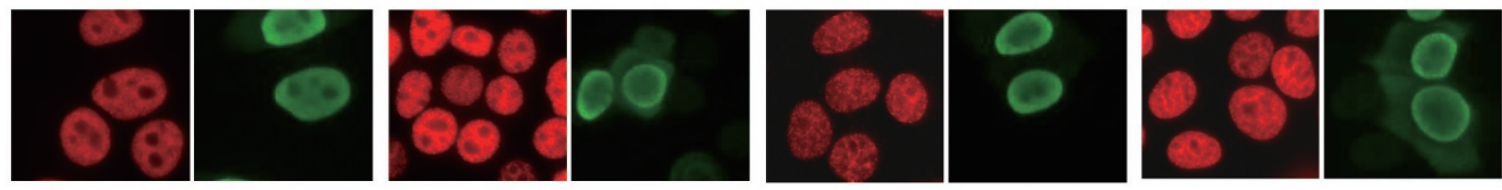

PHF8 (H247A)
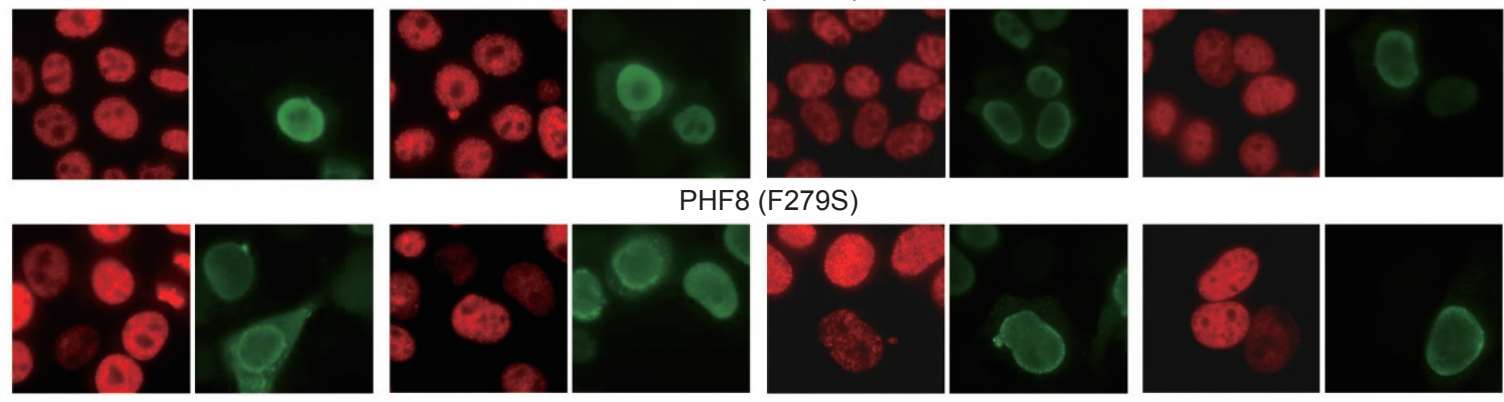

PHF8 ( $\triangle \mathrm{PHD})$

Figure 1 PHF8 controls H3K4 and H3K9 methylation in cells. (A) Diagram illustrating the structure of PHF8 and PHF8 mutants. (B) HeLa cells were transfected with Flag-tagged PHF8 or PHF8 mutants. The transfected cells were fixed, costained for expression of the proteins (anti-Flag, green) and the indicated histone modifications (red), and analyzed by a fluorescent microscope. 
tion states tested, we found that overexpression of FlagPHF 8 consistently led to reduced levels of H3K4me3, H3K4me2, H3K9me2 and H3K9me1 (Figure 1B), but had no significant effect on $\mathrm{H} 3 \mathrm{~K} 9 \mathrm{me} 3$ and $\mathrm{H} 3 \mathrm{~K} 4 \mathrm{me} 1$ (Figure 1C), H3K27me3/2/1 and H3K36me3/2 (Supplementary information, Figure S1). To test whether these activities are intrinsic to PHF8, we mutated a conserved histidine residue (H247) within the $\mathrm{JmjC}$ domain to alanine. The equivalent mutation has been shown to impair the histone demethylase activity of JMJD2A and other JmjC domain demethylases [18]. As shown in Figure 1B, this mutant is compromised for the demethylase activity. Together these results support PHF8 as a histone demethylase with dual $\mathrm{H} 3 \mathrm{~K} 4$ and $\mathrm{H} 3 \mathrm{~K} 9$ demethylase activity.

\section{PHF8 exhibits only $\mathrm{H} 3 \mathrm{~K} 9$ demethylase activity in vitro}

To test PHF8 demethylase activity in vitro, we expressed and purified Flag-PHF8 wild-type and H247A mutant from $293 \mathrm{~T}$ cells. As controls of substrate specificity, we also expressed and purified Flag-tagged JMJD1A and JMJD2A, which were previously shown to demethylate $\mathrm{H} 3 \mathrm{~K} 9 \mathrm{me} 2 / 1$ and $\mathrm{H} 3 \mathrm{~K} 9 \mathrm{me} 3 / 2$ and $\mathrm{H} 3 \mathrm{~K} 36 \mathrm{me} 2 / 1$, respectively [18]. These proteins were quantified by western blot analysis using the common Flag-tag (Figure $2 \mathrm{~A}$, lower panel), applied to in vitro demethylation reactions using core histones as substrates and subsequently analyzed by western blot. As shown in Figure 2A, the wild-type PHF 8 clearly exhibited H3K9me2 demethylase activity, whereas this activity was compromised for the H247A mutant. Consistent with previous data, JMJD1A was a demethylase specific for H3K9me2/1. Note that incubation with Flag-JMJD2A led to robust reduction of $\mathrm{H} 3 \mathrm{~K} 9 \mathrm{me} 3 / 2$ and a marked accumulation of $\mathrm{H} 3 \mathrm{~K} 9 \mathrm{me}$. This is consistent with previous findings that JMJD2A was able to demethylate $\mathrm{H} 3 \mathrm{~K} 9 \mathrm{me} 3 / 2$ but not H3K9me1. In this regard, despite the observed H3K9me2 demethylase activity for Flag-PHF8, no accumulation of H3K9me1 was observed, indicating that PHF8 also had the ability to remove $\mathrm{H} 3 \mathrm{~K} 9 \mathrm{me} 1$.

To our surprise, in this assay PHF8 did not exhibit
A
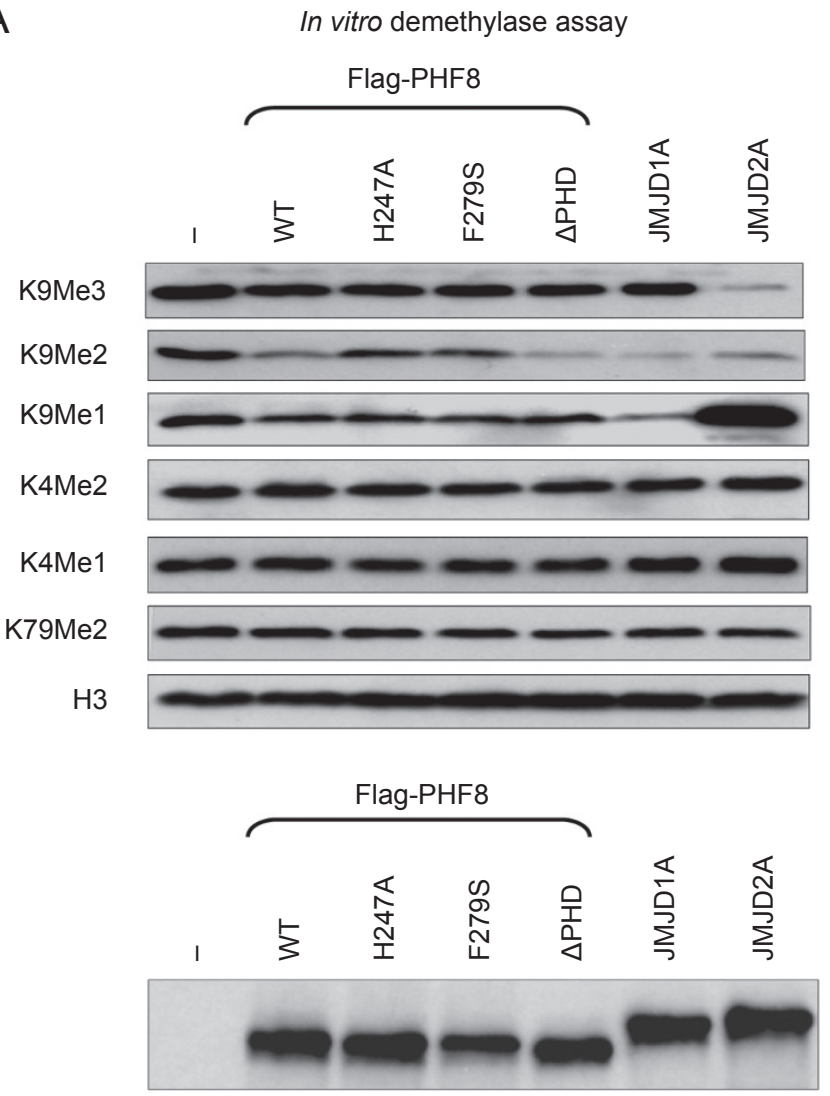

Western blot $(\alpha$ Flag $)$
B

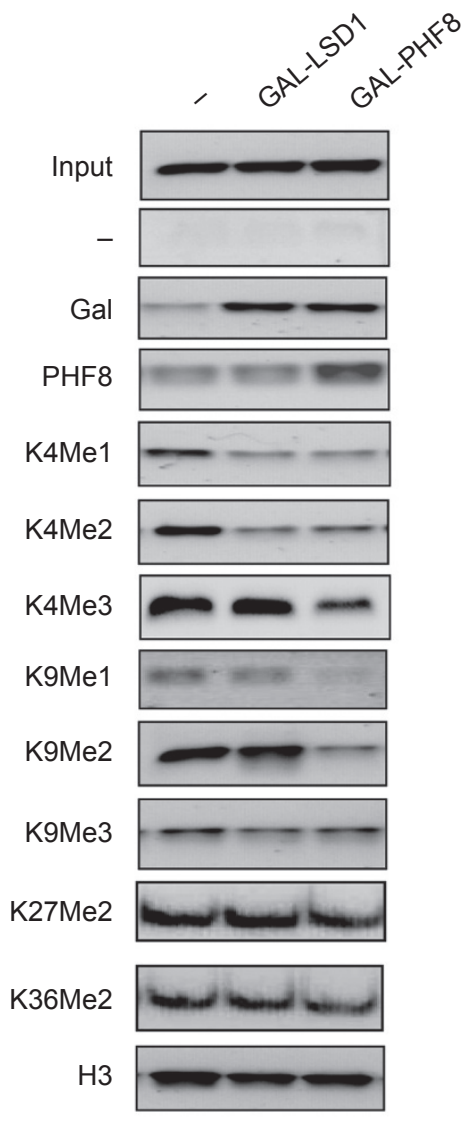

Figure 2 Histone demethylation by PHF8 in vitro and in Xenopus oocytes. (A) PHF8 exhibited only H3K9me2/1 demethylase activity in vitro. (B) In Xenopus oocytes PHF8 exhibited both H3K4 and K3K9 demethylase activity as revealed by ChIP analysis. 
any demethylase activity toward $\mathrm{H} 3 \mathrm{~K} 4 \mathrm{me} 2 / 1$. Under the same condition but in separate experiments, we also failed to observe any $\mathrm{H} 3 \mathrm{~K} 4 \mathrm{me} 3$ demethylase activity for PHF8 (Supplementary information, Figure S2). Thus, although PHF8 exhibits demethylase activity toward $\mathrm{H} 3 \mathrm{~K} 4 \mathrm{me} 3 / 2$ and $\mathrm{H} 3 \mathrm{~K} 9 \mathrm{me} 2 / 1$ in cells as shown above, purified PHF8 has only H3K9me2/1 demethylase activity in vitro.

\section{PHF8 exhibits both H3K4 and H3K9 demethylase activ-} ity in Xenopus oocytes

Given the discrepancy between the results from our in vitro demethylase assay and immunofluorescence, we next tested the demethylase activity of PHF 8 by chromatin immunoprecipitation (ChIP) in Xenopus oocytes, which is an excellent model system for studying histone modifications [19, 20]. We expressed PHF8 as a Gal4 DNA-binding domain fusion protein (Gal-PHF8) and assembled a reporter bearing four Gal4 binding sites $(4 \times$ UAS-TR $\beta A)$ into chromatin in Xenopus oocytes. As a control for H3K4 demethylation, we also expressed LSD1 as a Gal4 fusion (Gal-LSD1). As shown in Figure 2B, ChIP analysis confirmed the expression and binding of both Gal-LSD1 and Gal-PHF8 to the reporter.
Expression of either protein led to reduced levels of $\mathrm{H} 3 \mathrm{~K} 4 \mathrm{me} 2 / 1$; however, reduction of $\mathrm{H} 3 \mathrm{~K} 4 \mathrm{me} 3$ was only observed for Gal-PHF8. These results are in full agreement with reports showing that LSD1 is an $\mathrm{H} 3 \mathrm{~K} 4 \mathrm{me} 2 / 1$ specific demethylase [12]. Consistent with the H3K9me2 demethylase activity observed in our in vitro demethylation assay, expression of Gal-PHF8 also resulted in reduction of $\mathrm{H} 3 \mathrm{~K} 9 \mathrm{me} 2$ and $\mathrm{H} 3 \mathrm{~K} 9 \mathrm{me} 1$ but not H3K9me3. Furthermore, no significant effect on $\mathrm{H} 3 \mathrm{~K} 27 \mathrm{me} 2$ or H3K36me2 was observed for either Gal-PHF8 or GalLSD1. Taken together, results from both in vitro and in vivo demethylase assays support PHF8 as an H3K9me2/1 demethylase.

The mental retardation-linked F279S mutation impairs PHF8 histone demethylase activity

XLMR is a heterogeneous disease that affects around 1.6 in 1000 males, and the symptoms range from mild to severe mental retardation. PHF8 has recently been shown to be a causal gene for XLMR and cleft lip/cleft palate $[16,17]$. While most PHF8 mutations identified so far in patients are nonsense mutations that result in truncation of a large portion of the protein, one mutation (836T-C) changes phenylalanine at amino acid position 279 in the
A

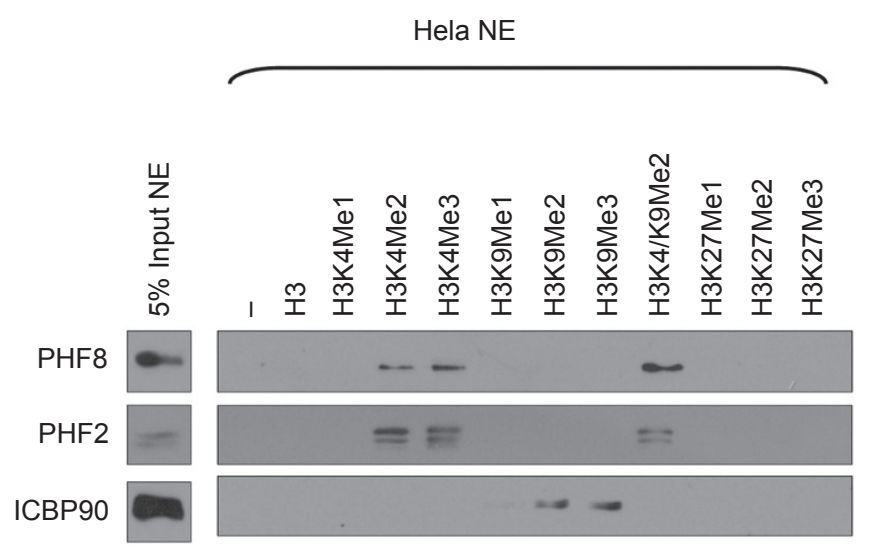

B

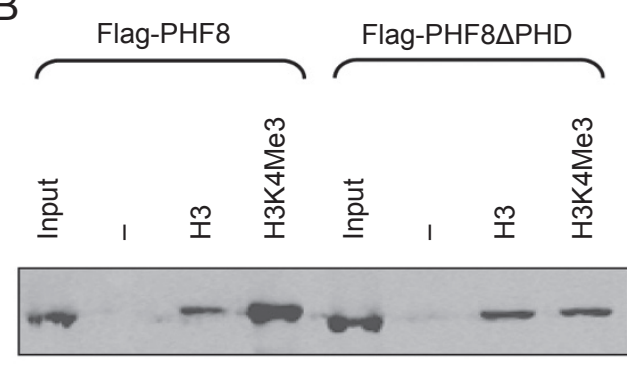

C

Recombinant His-PHF8 (1-428aa)

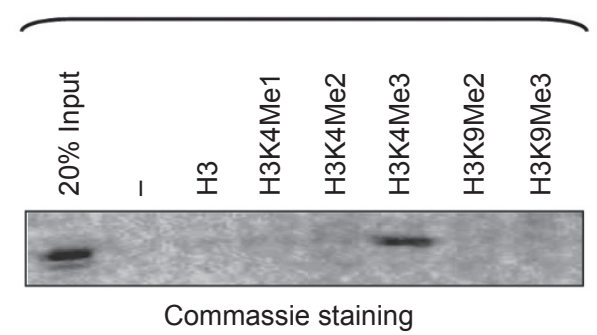

Figure 3 PHF8 specifically recognizes H3K4me3/2 modifications through its N-terminal PHD domain. (A) HeLa nuclear extracts were incubated with various C-terminal biotinylated histone $\mathrm{H} 3$ peptides (aa1-26, $1 \mu \mathrm{g}$ ) that were pre-bound to streptavidin-agarose beads. After extensive washing, the proteins associated with beads were separated by SDS-PAGE and followed by western blot analysis. (B) Flag-PHF8 and Flag-PHF8 PHD were expressed in HeLa cells by transient transfection and the resulting whole-cell extracts were used for histone tail peptide binding assay, followed by western blot analysis using an anti-Flag antibody. (C) His-PHF8(aa1-428) was expressed and purified from E. coli and applied to histone tail peptide binding assay. The binding of His-PHF8(aa1-428) was detected by Coomassie blue staining. 
JmjC domain to serine [17]. To test whether PHF8 enzymatic activity is causally linked to the disease, we examined whether the F279S mutation affects PHF8 enzymatic activity. Immunofluorescence experiments showed that expression of a Flag-tagged PHF8(F279S) mutant did not significantly affect the levels of $\mathrm{H} 3 \mathrm{~K} 4 \mathrm{me} 3 / 2$ and H3K9me2/1 (Figure 1B). Furthermore, the PHF8(F279S) mutant exhibited substantially reduced activity in vitro (Figure 2A). Together, these results demonstrate that the F279S mutation results in defect of PHF8 histone demethylase activity, suggesting a causal link between impairment of PHF8 histone demethylase activity and XLMR pathogenesis.

PHF8 recognizes $H 3 K 4 m e 3 / 2$ through an $N$-terminal PHD domain

Recent studies have defined the PHD domain as a module for binding histone tails [21]. The binding of methylated histones by PHD domains in various proteins has been linked to biological processes including transcription regulation and DNA repair. PHF8 contains an $\mathrm{N}$-terminal PHD domain. We thus analyzed whether PHF8 also recognizes or reads histone methylation and whether its PHD domain is involved. Using an in vitro binding assay, we found that PHF8 in HeLa nuclear extracts bound to $\mathrm{H} 3$ tail peptides with $\mathrm{K} 4 \mathrm{me} 2$ or $\mathrm{K} 4 \mathrm{me} 3$, but not to unmodified $\mathrm{H} 3$ peptide or $\mathrm{H} 3 \mathrm{~K} 9$ methylated peptides. As a control of binding specificity [22], ICBP90/ UHRF 1 bound to $\mathrm{H} 3$ peptides with $\mathrm{K} 9 \mathrm{me} 3 / 2$ but not with $\mathrm{K} 4 \mathrm{me} 3 / 2$. The PHD domain mediates the binding of PHF8 to H3K4me3/2, as a PHD domain deletion mutant did not show any preference for the H3K4me3 peptide (Figure 3B). The binding of H3K4me3 by PHF8 is likely direct, as purified recombinant PHF8 fragment aa1-428, which includes the PHD domain, bound to the H3K4me3 peptide (Figure 3C).

We next analyzed if the PHD domain plays a role in histone demethylation by PHF8. Immunofluorescence experiments in Figure 1B showed that deletion of the PHD domain has no effect on PHF8 demethylase activity. The result in Figure 2A also showed that PHD domain is not essential for PHF8 demethylase activity in vitro. Collectively these results indicate that although PHF8 recognizes $\mathrm{H} 3 \mathrm{~K} 4 \mathrm{me} 3 / 2$ epigenetic marks, this activity appears to be not essential for its histone demethylase activity.

Knockdown of PHF8 impairs RA-induced neuronal differentiation and overexpression of wild-type but not F279S mutant results in neurogenesis in P19 cells

The fact that PHF8 mutations result in XLMR suggests a role for PHF8 in neuronal differentiation. Mouse
P19 embryonic carcinoma cells are a well-characterized model for in vitro neuronal differentiation, which upon exposure to RA differentiate into neurons [23]. To determine the role of PHF8 in neuron differentiation, we knocked down PHF8 in P19 cells by two different PHF8specific siRNAs (siPHF8-A and siPHF8-B) and examined their effect on RA-induced neuronal differentiation. RA-induced neuronal differentiation can be scored by loss of the pluriopotency marker Oct4, appearance of neuronal marker class III $\beta$-tubulin (Tuj1) and neuroncharacteristic morphology. We established the condition that treatment with both siRNAs resulted in $\sim 60-75 \%$ reduction of PHF8 proteins in comparison to treatment with a control siRNA (Figure 4A). The results in Figure $4 \mathrm{C}$ show that both siPHF8-treated cells exhibited a marked reduction of Tuj1-positive neuronal cells and a concomitant increase of Oct4-positive cells. Note that the Tuj1-positive cells are of typical neuronal cell morphology. Similar results were observed in three independent experiments and quantitative results from one representative experiment are shown in Figure 4B.

To further characterize the role of PHF 8 and its demethylase activity in neuronal differentiation, we established pools of P19 cells stably transfected with Flag-PHF8 or Flag-PHF8(F279S) mutant. The overexpression of Flag-PHF8 and Flag-PHF8(F279S) was confirmed by western blot analysis using anti-PHF8 antibody (Figure 4D). We found by immunostaining that many Flag-PHF8-expressing cells were positive for neuronal cell marker Tuj1 (Figure 4F and quantitative results in Figure 4B). In contrast, no increased Tuj1positive cells were detected in the Flag-PHF8(F279S)expressing cells, suggesting that PHF8 overexpression could drive P19 into neurogenesis in a demethylasedependent manner. We confirmed this result by RT-PCR analysis of a panel of neuronal markers including Tuj1, GFAP, nestin, NeuroD, neurogenin1 and PAX6 [24]. As shown in Figure 4E, all these markers were up-regulated in the Flag-PHF8 but not in the Flag-PHF8(F279S) cells. Consistent with a neuronal differentiation phenotype, the expression of stem cell marker Oct4 decreased in the Flag-PHF8 cells. Together these results support an important role of PHF8 in neuronal differentiation.

\section{PHF8 interacts with liganded RAR and functions as an RAR coactivator}

RA exerts its neuronal differentiation inducer function through RARs, a member of the nuclear receptor superfamily [25]. Given that PHF8 is a histone demethylase required for RA-induced neuronal differentiation, we next tested whether a YFP-tagged PHF8 interacts with CFP-Lac-RAR $\alpha$ in A03_1 CHO DG44 cells, in which 
A

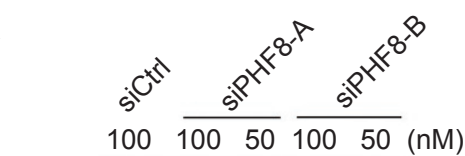

aPHF8

$\alpha$-actin

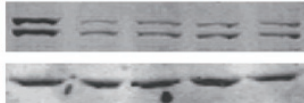

D

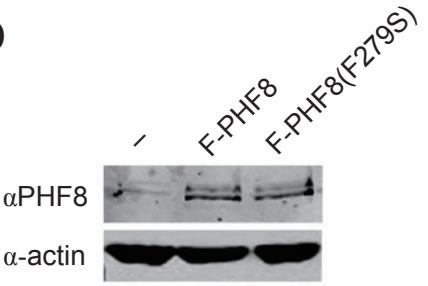

B

\begin{tabular}{|l|c|c|c|c|c|}
\hline & TUJ1 & OCT4 & DAPI & TUJ1 \% & OCT4 \% \\
\hline- & 263 & 131 & 431 & $61.02 \%$ & $30.39 \%$ \\
\hline siCtrl & 217 & 143 & 372 & $58.33 \%$ & $38.44 \%$ \\
\hline siPHF8-A & 108 & 412 & 559 & $19.32 \%$ & $73.70 \%$ \\
\hline siPHF8-B & 69 & 542 & 633 & $10.90 \%$ & $85.62 \%$ \\
\hline Flag-PHF8 & 151 & 287 & 445 & $33.93 \%$ & $64.49 \%$ \\
\hline Flag-PHF8(F279S) & 2 & 411 & 409 & $0.49 \%$ & $99.51 \%$ \\
\hline
\end{tabular}

$E$

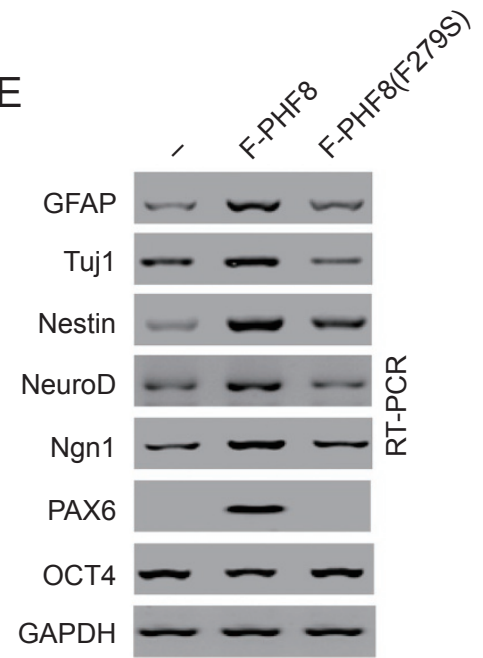

C
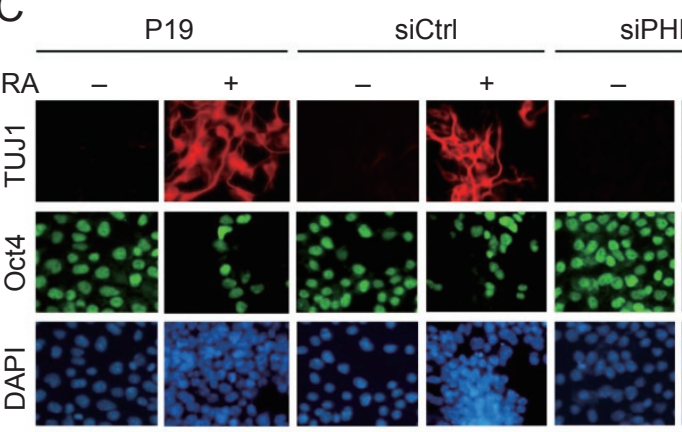

iPHF8-A

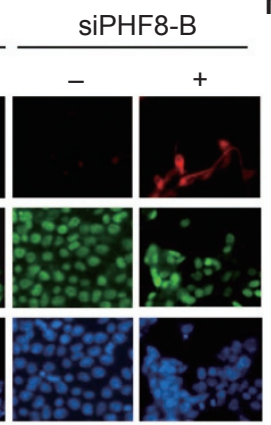

$\mathrm{F}$

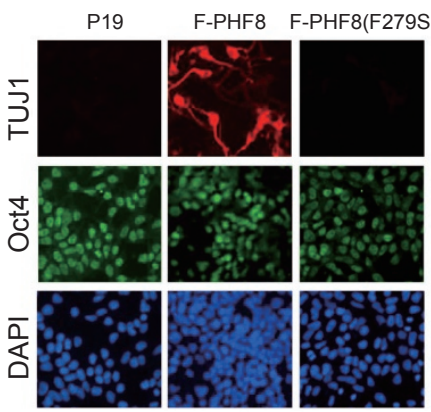

Figure 4 PHF8 regulates neuronal differentiation in mouse embryonic carcinoma P19 cells. (A) P19 cells were treated with siRNAs (100 nM) as indicated for 2 days and then analyzed for levels of PHF8 protein by western blot. (B) Quantitative results of Tuj1 and Oct4-positive cells from experiments in $\mathbf{C}$ and $\mathbf{E}$ (three independent experiments). (C) P19 cells were treated with or without siRNA as indicated for 2 days and then subjected to $10^{-6} \mathrm{M}$ RA-induced neuronal differentiation protocol. In the end, the cells were stained for the neuronal marker class III $\beta$-tubulin (Tuj1), the pluriopotency marker Oct4 (undifferentiated) and DAPI to reveal the nuclei of all cells. (D) Stable overexpression of Flag-PHF8 and Flag-PHF8(F279S) mutant in P19 cells as revealed by western blot. (E) RT-PCR analysis of a panel of neuronal cell markers. GAPDH served as a control. (F) Immunostaining showing Tuj1- or Oct4-positive cells. Note the appearance of neuronal cell morphology for Tuj1-positive cells.

binding of CFP-Lac-RAR $\alpha$ to a region containing integrated tens of thousands of direct repeats of the lac operator can be observed (Figure 5A) [26]. The results in Figure 5B show that YFP-PHF8 was colocalized with bright CFP-Lac-RAR $\alpha$ foci only upon RA treatment. After counting a large number of cells we observed a complete co-localization of these two proteins in $\sim 65 \%$ of the cells, partial colocalization in $\sim 20 \%$ of the cells and no colocalization in $\sim 15 \%$ of the cells. These results indicate that PHF8 interacts with RAR $\alpha$ in an RA-dependent manner.

We next analyzed whether PHF8 modulates transcriptional activity of RAR $\alpha$. In transient transfection assays, we found that PHF8, but not the enzymatic deficient
H247A mutant, enhanced transcription of a RAR-driven luciferase reporter (Figure 5D), indicating that the demethylase activity of PHF8 is required for its coactivator function. PHF 8 also mildly enhanced RAR transcriptional activity in the absence of RA treatment, presumably due to the presence of residual RA in the culture medium.

We also carried out ChIP assays to test whether PHF8 was targeted to three known endogenous RAR target genes, namely HOXA1, HOXB1 and RAR $\beta 2$ in P19 cells. P19 cells were treated with or without RA for 6 $\mathrm{h}$ and processed for ChIP assays. The results in Figure 5E showed that PHF8 was indeed recruited to all three genes upon $6 \mathrm{~h}$ of RA treatment. The specificity of 
A

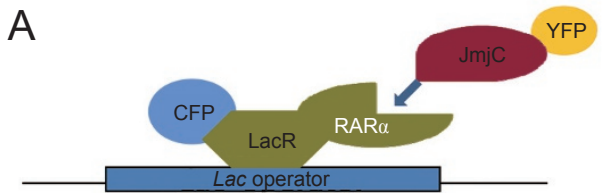

B

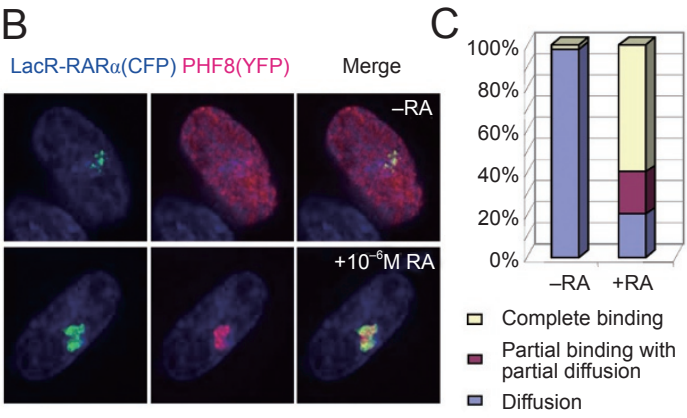

D

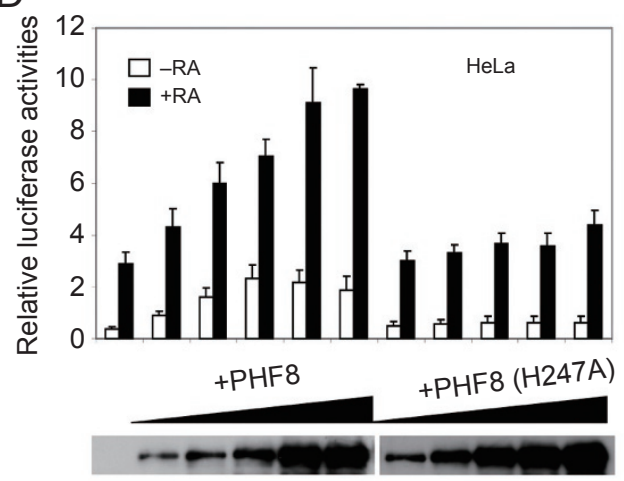

E

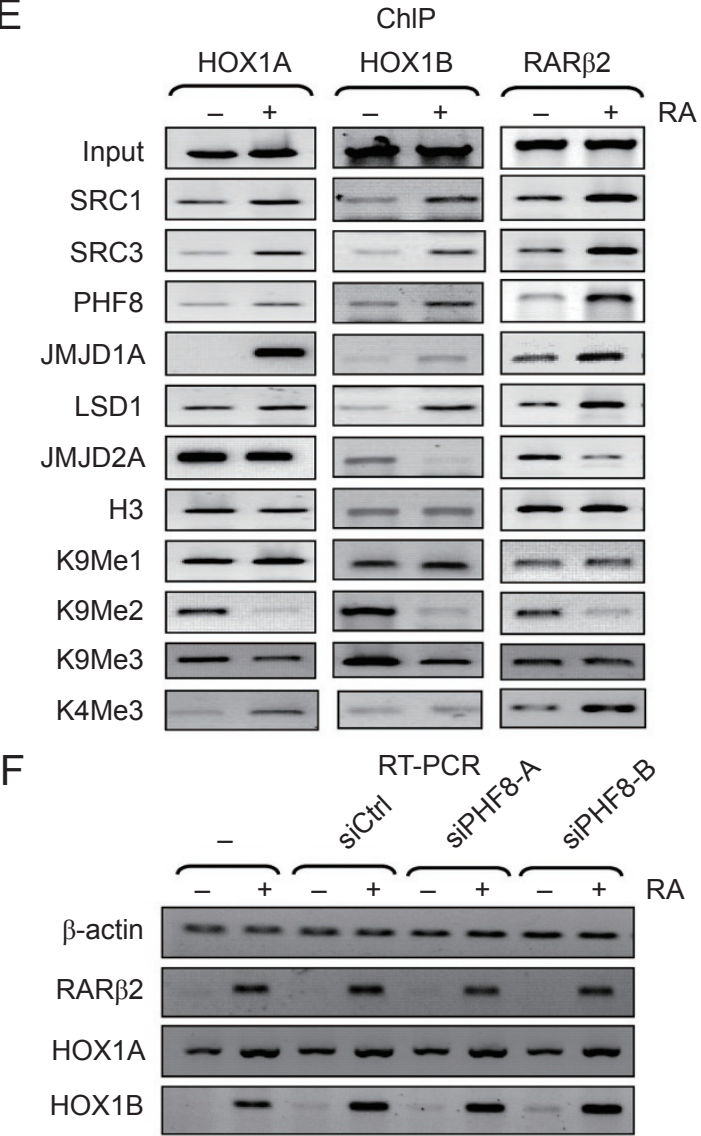

Figure 5 PHF8 is a transcriptional coactivator for RAR $\alpha$. (A) Diagram illustrating assay for RAR $\alpha$ and PHF8 interaction in A03_1 cells. (B) A03_1 cells co-transfected with YFP-PHF8 and CFP-LacR-RAR $\alpha$ were subjected to deconvolution microscopy analysis. Nuclei were visualized by DNA staining with DAPI (blue). (C) Percent of cells (Y-axis) that co-transfected with YFP-PHF8 and CFP-LacR-RAR $\alpha$ and showed complete binding (tan), partial binding with partial diffusion (purple) and diffusion (blue) upon treatment with or without all-trans retinoic acid (RA, at $10^{-6} \mathrm{M}$ ) for $3 \mathrm{~h}$. (D) HeLa cells were co-transfected with $125 \mathrm{ng}$ of Gal4-RAR $\alpha$ expression vector, $125 \mathrm{ng}$ of $4 \times$ UAS-TK-luc reporter gene, various amounts of PHF8 vector or PHF8(H247A) in the absence or presence of RA at $10^{-6} \mathrm{M}$. The western blot analysis with antibody against Flag was performed by using the whole cell extracts from the luciferase experiments (the bottom panel). The relative luciferase activities are the mean \pm SEM of three independent transfections normalized to $\beta$-galactosidase activity. (E) P19 cells were treated with or without $10^{-6} \mathrm{M}$ RA for $6 \mathrm{~h}$ and then proceeded for ChIP analysis for the promoter regions of three RAR target genes using antibodies as indicated. (F) P19 cells were treated with or without the indicated siRNAs for 2 days followed by $10^{-6} \mathrm{M}$ RA for $6 \mathrm{~h}$. RT-PCR for three RAR target genes was performed. RT-PCR for $\beta$-actin served as a control.

PHF8 antibody was confirmed as siPHF8-B treatment led to essentially loss of ChIP signal for PHF8 (Supplementary information, Figure S4). In addition to PHF8, our ChIP analysis also revealed the RA-induced recruitment of coactivators SRC-1 and SRC-3 and two additional histone demethylases JMJD1A and LSD1. On the contrary, RA treatment resulted in dissociation of demethylase JMJD2A. Analysis of histone modifications showed that RA treatment in general resulted in increase in $\mathrm{H} 3 \mathrm{~K} 9 \mathrm{me} 1$ and $\mathrm{H} 3 \mathrm{~K} 4 \mathrm{me} 3$ and decrease in $\mathrm{H} 3 \mathrm{~K} 9 \mathrm{me} 2 / 3$, among these changes the decrease in $\mathrm{H} 3 \mathrm{~K} 9 \mathrm{me} 2$ is most prominent.

Interestingly, we found that knockdown of PHF 8 did not significantly affect RA-induced transcriptional activation of the HOXA1, HOXB1 and RAR $\beta 2$ genes as shown by RT-PCR (Figure 5F). The knockdown of PHF 8 by both PHF 8 siRNAs was confirmed by western blot analysis (Supplementary information, Figure S5). The subsequent ChIP analysis for the HOXB1 promoter confirmed the effective knockdown of PHF8 and insignificant effect on RA-induced reduction of H3K9me2 (Supplementary information, Figure S4). 


\section{Discussion}

Mutation of PHF 8 has been linked with onset of XLMR with cleft lip/cleft palate [16, 17, 27]. In this study we show that PHF8 is a histone demethylase that may play an important role in neuronal differentiation. The most important findings in this study are: (1) the identification of PHF8 as a histone demethylase; (2) the disease-linked F279S mutation impairs the PHF8 demethylase activity; (3) PHF8 overexpression drives neurogenesis and knockdown of PHF8 impairs RA-induced neuronal differentiation in the mouse P19 model; and (4) PHF8 is recruited to endogenous RAR target genes and functions as a RAR coactivator in a demethylase activity-dependent manner in transient transfection.

Mammalian PHF 8 belongs to a subfamily of JmjC domain- and PHD domain-containing proteins, which also includes PHF2 and KIAA1718/KDM7A. While a PHF2 homolog is present in $S$. pombe, zebrafish contains a homolog for PHF2 and PHF 8. During preparation and revision of our manuscript, a few papers reported the structure, enzymatic activity and function of this subfamily of proteins. Based on in vitro demethylation assays Loenarz et al. first reported that PHF 8 catalyzed demethylation of $\mathrm{H} 3 \mathrm{~K} 9 \mathrm{me} 2 / 1$, H3K27me2 and H3K36me2 [28]. Other studies show PHF 8 as a predominant $\mathrm{H} 3 \mathrm{~K} 9 \mathrm{me} 2 / 1$ demethylase with either minimal H3K27me2 and H3K36me2 demethylase activity [29] or $\mathrm{H} 3 \mathrm{~K} 9 \mathrm{me} 2 / 1$ demethylase activity only [30-33]. In agreement with the latter studies, we showed that in all assays PHF8 exhibited histone demethylase activity with $\mathrm{H} 3 \mathrm{~K} 9 \mathrm{me} 2 / 1$ specificity. In this regard, PHF2 was reported to be an H3K9me1 demethylase [34], whereas KIAA1718/KDM7A exhibits a dual H3K9 and H3K27 demethylase activity [35].

Interestingly, we found that PHF 8 also exhibits a H3K4 demethylase activity both in HeLa cells and Xenopus oocytes. Given the lack of this enzymatic activity for purified PHF8 in vitro (Figure 2A) [28, 29], we suggest that the H3K4 enzymatic specificity of PHF8 may be dependent on one or more cellular proteins that dissociated from PHF8 upon purification. However, we could not rule out the possibility that the observed H3K4 demethylase activity might be an indirect effect of PHF8 or even the artifact of PHF8 overexpression.

The PHD finger has recently emerged as a module that binds to or "reads" methylated or unmethylated histones [7, 21]. Importantly, mutations in PHD fingers and consequent misinterpretation of histone codes have been linked with human pathologies $[21,36]$. We show that PHF 8 also specifically recognizes $\mathrm{H} 3 \mathrm{~K} 4 \mathrm{me} 3 / 2$ and this activity requires its PHD finger. In this regard,
Horton et al. reported that the presence of PHD domain enhances $\mathrm{H} 3 \mathrm{~K} 9 \mathrm{me} 2 / 1$ demethylase activity toward the H3K9me2 peptide that also contains K4me3 [29], suggesting a role of binding $\mathrm{H} 3 \mathrm{~K} 4 \mathrm{me} 2 / 3$ in targeting PHF8 for H3K9me2/1 demethylation. However, in our experiments, the PHD domain seems not required for PHF8 histone demethylase activity both in vitro and in cells (Figures 1 and 2). This discrepancy likely suggests that in cells and the core histone substrates used in our in vitro assay the majority of $\mathrm{H} 3$ proteins do not contain both $\mathrm{K} 4 \mathrm{me} 3 / 2$ and $\mathrm{K} 9 \mathrm{me} 2$. It is also noteworthy that PHF8 is associated with mitotic chromosomes in HeLa cells (Supplementary information, Figure S2) and this association is not altered with deletion of PHD finger.

The mental retardation in patients with PHF 8 mutations implies a role of PHF8 in neurogenesis and/or neuronal differentiation. In others' and our studies, F279S mutation was shown to impair the PHF8 demethylase activity, thus linking its demethylase activity defect with the onset of mental retardation. In support of a role in neurogenesis and/or neuronal differentiation, we showed that PHF8 is required for RA-induced differentiation of mouse P19 cells toward neuronal cells, because knockdown of PHF8 in P19 cells with two different siRNAs against PHF8 all significantly impaired RA-induced neuronal differentiation (Figure 4). Furthermore, we showed that overexpression of PHF8 in P19 cells drives neurogenesis. This effect is dependent on its demethylase activity and is likely associated with its ability to induce the expression of PAX6, NeuroD and neurogenin 1, the key transcription factors in neurogenesis. How PHF8 regulates PAX6, NeuroD and neurogenin 1 remains to be investigated. In this regard, it is noteworthy that KIAA1718/KDM7A is also shown to regulate neural differentiation in a histone demethylase-dependent manner [35]. It is of interest in future to determine the relationship and relative roles of PHF8 and KIAA1718/ KDM7A in neurogenesis and differentiation.

In an effort to understand how PHF8 influences RAinduced neuronal differentiation, we found that PHF8 interacts with RAR $\alpha$ in a ligand-dependent manner in cells (Figure 5B) and functions in transient transfection as an RAR coactivator in a histone demethylase activitydependent manner (Figure 5C). The interaction between PHF 8 and RAR $\alpha$ is further supported by in vitro pulldown and coimmunoprecipitation experiments (Supplementary information, Figure S6), although in these in vitro assays the interaction between these two proteins is by and large RA-independent. Despite the recruitment of PHF8 to all three RAR target genes tested (Figure 5E), knockdown of PHF8 did not appear to significantly affect their RA-induced transcriptional activation (Figure 5F), 
perhaps due to the involvement of other demethylases such as JMJD1A and LSD1. It is possible that PHF8 influences RA-induced neuronal differentiation in P19 cells by affecting RAR target genes yet to be tested here or genes induced at the later stages of neuronal differentiation.

Interestingly, PHF2, PHF8 and KIAA1718/KDM7A are all nuclear proteins with enriched nucleolar localization $[30,34,35]$. Consistent with this feature, both PHF2 and PHF8 have been shown to associate with rDNA promoters and regulate rDNA transcription. It remains to be determined if mis-regulation of rDNA regulation is associated with the disease onset of XLMR patients bearing PHF8 mutations.

In sum, we show here that PHF 8 is a histone demethylase and coactivator for RAR $\alpha$ and that the F279S mutation identified in an XLMR patient results in loss of its demethylase activity. In this regard, JARID1A, a JmjC domain protein whose mutations are also causally linked with XLMR, and its related proteins have recently been identified as H3K4 demethylases [37, 38]. Together these results indicate that appropriate control of histone methylation is critically important for correct cellular identity and differentiation in mammalian development.

\section{Materials and Methods}

\section{Cell culture, reagents and plasmids}

HeLa cells were routinely maintained in Opti-MEM I media (Invitrogen) with $4 \%$ fetal bovine serum. A03_1 cells were maintained in customized F-12 Ham's media with $0.3 \mu \mathrm{M}$ methotrexate and $10 \%$ dialyzed fetal bovine serum. Mouse embryonic carcinoma cells (P19) were cultured in DMEM/F12 (1:1) supplemented with $10 \%$ fetal calf serum. Stable Flag-PHF8 and FlagPHF8(F279S) P19 cells were generated by transfection followed by 2 -week selection with puromycin. The antibodies used were listed in Supplementary information, Table S1.

The plasmids for CFP-LacR-tagged RAR $\alpha$, RAR $\alpha$ and the RAR $\beta 2$ promoter-driven luciferase reporter have been described previously [26, 39]. All PHF8 mutants were generated by PCRbased site-directed mutagenesis or cloning. All plasmid constructs were verified by DNA sequencing.

\section{Luciferase and western blot assays}

Transient transfection-based luciferase assay in HeLa cells was performed essentially as described previously [39]. Western blot analysis was performed essentially as described [39].

\footnotetext{
Indirect immunofluorescence and deconvolution microscopy

Immunostaining experiments with HeLa cells were performed essentially as described [40]. Images were acquired with a Zeiss deconvolution microscope. The transfection of A03 1 cells with CFP and YFP-tagged plasmids and deconvolution microscopy were essentially performed using GeneJuice (Novagen) as described [26].
}

In vitro demethylase assay

Flag-tagged proteins were purified with anti-Flag M2 Affinity Gel (Sigma) and used for in vitro demethylation as described [13].

\section{Histone tail peptide pull-down assay}

Histone tail peptide pull-down was essentially performed as described [22].

In vitro $m R N A$ preparation, microinjection of Xenopus oocytes and ChIP assay

In vitro synthesis of Gal-PHF8 and Gal-LSD1 mRNA was carried out using linearized pSP64poly(A)-Gal4-PHF8 (Afl II digestion) and pSP64poly(A)-Gal4-LSD1 and a SP6 Message Machine kit from Ambion according to the manufacturer's instructions. Preparation and microinjection of stage VI Xenopus oocytes with mRNA and reporter DNA and ChIP were essentially performed as described [20].

\section{siRNA treatment, RT-PCR, ChIP and RA induction of neuronal differentiation}

The siPHF8-A and siPHF8-B were synthesized by GenePharma and the sequences are listed in Supplementary information, Table $\mathrm{S} 1$. The transfection (100 nM siRNA) was performed with a seeding density of $3 \times 10^{5}$ cell $/$ dish $(6 \mathrm{~cm})$ using Lipofectamine transfection reagents (Invitrogen) following the supplier's protocol.

To analyze the effect of PHF8 knockdown on RA-induced gene expression, P19 cells were transfected with or without siRNAs as indicated for $60 \mathrm{~h}$. Then P19 cells were treated with or without $10^{-6} \mathrm{M}$ RA for $6 \mathrm{~h}$ and collected for RT-PCR or ChIP as described [24] using the primers listed in Supplementary information, Table S1. RA-induced P19 neuronal differentiation of P19 cells was performed essentially as described [24].

\section{Acknowledgment}

We thank Drs Paul Wade (National Institute of Environmental Health Sciences, USA) and David Stewart (University of Houston, USA) for their critical reading of the manuscript. We also thank Dr Naihe Jing (Institute of Biochemistry and Cell Biology, SIBS, China) for assistance in RA-induced neuronal differentiation of P19 cells. This study was supported by grants from the National Natural Science Foundation of China (90919025, 30871381), the Ministry of Science and Technology of China (2009CB918402, 2009CB825601), and by funds from Ladies Leukemia League (LLL) (to SD), DLDCC Cancer Biology Pilot Project Award (to SD) and the Albert and Margaret Alkek Foundation (to SD).

\section{References}

1 Margueron R, Trojer P, Reinberg D. The key to development: interpreting the histone code? Curr Opin Genet Dev 2005; 15:163-176.

2 Roeder RG. Transcriptional regulation and the role of diverse coactivators in animal cells. FEBS Lett 2005; 579:909-915.

3 Wang GG, Allis CD, Chi P. Chromatin remodeling and cancer, Part I: covalent histone modifications. Trends Mol Med 2007; 13:363-372.

4 Martin C, Zhang Y. The diverse functions of histone lysine 
methylation. Nat Rev Mol Cell Biol 2005; 6:838-849.

5 Dillon SC, Zhang X, Trievel RC, Cheng X. The SET-domain protein superfamily: protein lysine methyltransferases. Genome Biol 2005; 6:227.

6 Vermeulen M, Mulder KW, Denissov S, et al. Selective anchoring of TFIID to nucleosomes by trimethylation of histone H3 lysine 4. Cell 2007; 131:58-69.

7 Wysocka J, Swigut T, Xiao H, et al. A PHD finger of NURF couples histone H3 lysine 4 trimethylation with chromatin remodelling. Nature 2006; 442:86-90.

8 Nishioka K, Chuikov S, Sarma K, et al. Set9, a novel histone H3 methyltransferase that facilitates transcription by precluding histone tail modifications required for heterochromatin formation. Genes Dev 2002; 16:479-489.

9 Zegerman P, Canas B, Pappin D, Kouzarides T. Histone H3 lysine 4 methylation disrupts binding of nucleosome remodeling and deacetylase (NuRD) repressor complex. J Biol Chem 2002; 277:11621-11624.

10 Bannister AJ, Zegerman P, Partridge JF, et al. Selective recognition of methylated lysine 9 on histone H3 by the HP1 chromo domain. Nature 2001; 410:120-124.

11 Lachner M, O'Carroll D, Rea S, Mechtler K, Jenuwein T. Methylation of histone H3 lysine 9 creates a binding site for HP1 proteins. Nature 2001; 410:116-120.

12 Shi Y, Lan F, Matson C, et al. Histone demethylation mediated by the nuclear amine oxidase homolog LSD1. Cell 2004; 119:941-953.

13 Tsukada Y, Fang J, Erdjument-Bromage H, et al. Histone demethylation by a family of JmjC domain-containing proteins. Nature 2006; 439:811-816.

14 Culhane JC, Cole PA. LSD1 and the chemistry of histone demethylation. Curr Opin Chem Biol 2007; 11:561-568.

15 Schneider J, Shilatifard A. Histone demethylation by hydroxylation: chemistry in action. ACS Chem Biol 2006; 1:75-81.

16 Laumonnier F, Holbert S, Ronce N, et al. Mutations in PHF8 are associated with $\mathrm{X}$ linked mental retardation and cleft lip/ cleft palate. J Med Genet 2005; 42:780-786.

17 Koivisto AM, Ala-Mello S, Lemmela S, et al. Screening of mutations in the PHF8 gene and identification of a novel mutation in a Finnish family with XLMR and cleft lip/cleft palate. Clin Genet 2007; 72:145-149.

18 Klose RJ, Yamane K, Bae Y, et al. The transcriptional repressor JHDM3A demethylates trimethyl histone H3 lysine 9 and lysine 36. Nature 2006; 442:312-316.

19 Almouzni G, Wolffe AP. Replication-coupled chromatin assembly is required for the repression of basal transcription in vivo. Genes Dev 1993; 7:2033-2047.

20 Stewart MD, Li J, Wong J. Relationship between histone H3 lysine 9 methylation, transcription repression, and heterochromatin protein 1 recruitment. Mol Cell Biol 2005; 25:25252538.

21 Baker LA, Allis CD, Wang GG. PHD fingers in human diseases: disorders arising from misinterpreting epigenetic marks. Mutat Res 2008; 647:3-12.

22 Karagianni P, Amazit L, Qin J, Wong J. ICBP90, a novel methyl K9 H3 binding protein linking protein ubiquitination with heterochromatin formation. Mol Cell Biol 2008; 28:705717.

23 Soprano DR, Teets BW, Soprano KJ. Role of retinoic acid in the differentiation of embryonal carcinoma and embryonic stem cells. Vitam Horm 2007; 75:69-95.

24 Kim S, Yoon YS, Kim JW, et al. Neurogenin1 is sufficient to induce neuronal differentiation of embryonal carcinoma P19 cells in the absence of retinoic acid. Cell Mol Neurobiol 2004; 24:343-356.

25 Mangelsdorf DJ, Thummel C, Beato M, et al. The nuclear receptor superfamily: the second decade. Cell 1995; 83:835839.

26 Qiu J, Huang Y, Chen G, et al. Aberrant chromatin remodeling by retinoic acid receptor alpha fusion proteins assessed at the single-cell level. Mol Biol Cell 2007; 18:3941-3951.

27 Abidi FE, Miano MG, Murray JC, Schwartz CE. A novel mutation in the PHF8 gene is associated with X-linked mental retardation with cleft lip/cleft palate. Clin Genet 2007; 72:1922.

28 Loenarz C, Ge W, Coleman ML, et al. PHF8, a gene associated with cleft lip/palate and mental retardation, encodes for an Nepsilon-dimethyl lysine demethylase. Hum Mol Genet 2010; 19:217-222.

29 Horton JR, Upadhyay AK, Qi HH, et al. Enzymatic and structural insights for substrate specificity of a family of jumonji histone lysine demethylases. Nat Struct Mol Biol 2010; 17:3843.

30 Feng W, Yonezawa M, Ye J, Jenuwein T, Grummt I. PHF8 activates transcription of rRNA genes through $\mathrm{H} 3 \mathrm{~K} 4 \mathrm{me} 3$ binding and H3K9me1/2 demethylation. Nat Struct Mol Biol 2010; 17:445-450.

31 Fortschegger K, de Graaf P, Outchkourov NS, et al. PHF8 targets histone methylation and RNA polymerase II to activate transcription. Mol Cell Biol 2010 Apr 26; doi:10.1128/ MCB.01520-09.

32 Kleine-Kohlbrecher D, Christensen J, Vandamme J, et al. A functional link between the histone demethylase PHF8 and the transcription factor ZNF711 in X-linked mental retardation. Mol Cell 2010; 38:165-178.

33 Yu L, Wang Y, Huang S, et al. Structural insights into a novel histone demethylase PHF8. Cell Res 2010; 20:166-173.

34 Wen H, Li J, Song T, et al. Recognition of histone H3K4 trimethylation by the plant homeodomain of PHF2 modulates histone demethylation. J Biol Chem 2010; 285:9322-9326.

35 Huang C, Xiang Y, Wang Y, et al. Dual-specificity histone demethylase KIAA1718 (KDM7A) regulates neural differentiation through FGF4. Cell Res 2010; 20:154-165.

36 Wang GG, Song J, Wang Z, et al. Haematopoietic malignancies caused by dysregulation of a chromatin-binding PHD finger. Nature 2009; 459:847-851.

37 Christensen J, Agger K, Cloos PA, et al. RBP2 belongs to a family of demethylases, specific for tri-and dimethylated lysine 4 on histone 3. Cell 2007; 128:1063-1076.

38 Klose RJ, Yan Q, Tothova Z, et al. The retinoblastoma binding protein RBP2 is an H3K4 demethylase. Cell 2007; 128:889900.

39 Dong S, Tweardy DJ. Interactions of STAT5b-RARalpha, a novel acute promyelocytic leukemia fusion protein, with retinoic acid receptor and STAT3 signaling pathways. Blood 2002; 99:2637-2646.

40 Qiu J, Wong J, Tweardy DJ, Dong S. Decreased intranuclear mobility of acute myeloid leukemia 1-containing fusion pro- 
teins is accompanied by reduced mobility and compartmentalization of core binding factor beta. Oncogene 2006; 25:39823993.
(Supplementary information is linked to the online version of the paper on Cell Research website.) 